\title{
Cerebral venous and sinus thrombosis with complicating thromboangiitis obliterans
}

\author{
Stefan Einsiedler MD, Georg Hödl MD, Raffi Topakian MD
}

- Cite as: CMAJ 2021 March 1;193:E311. doi: 10.1503/cmaj.201166

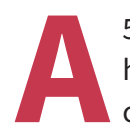

51-year-old man presented to our hospital with new onset of headache, nausea and blurred vision over the previous 4 days. A current smoker, his history included thromboangiitis obliterans (also known as Buerger disease) that resulted in left transtibial amputation. The patient's only medication was acetylsalicylic acid $100 \mathrm{mg} / \mathrm{d}$. On examination, the patient had bilateral papilloedema, but no focal neurologic deficit. Computed tomography (CT) venography showed cerebral venous sinus thrombosis (CVST) (Figure 1). Work-up was unremarkable for infectious, autoimmune, neoplastic and thrombophilic disorders, other than thromboangiitis obliterans. Our patient's symptoms resolved within a week after therapeutic anticoagulation with dalteparin, though he had persistent papilloedema. Repeat neuroimaging before switching to oral anticoagulation excluded secondary venous hemorrhage, despite persistent extensive CVST (Figure 2).

Cerebral venous sinus thrombosis accounts for approximately $1 \%$ of strokes, with an incidence of 5 people per million annually. ${ }^{1}$ Eighty percent of cases occur in people younger than 50 , with risk factors extending across a full spectrum of acquired and genetic thrombophilias. ${ }^{1}$ Patients commonly present with headache and worsening vision, although some develop focal neurologic deficits, seizures or alterations of consciousness. The combination of headaches and papilloedema, especially in someone with known thrombophilia, should increase the index of suspicion for CVST. The diagnosis can be missed when neuroimaging is ordered without venography; this might be the case for patients with insidious onset of headache and no other symptoms or signs. Anatomic variability of the venous sinuses makes CT insufficiently sensitive to diagnose CVST, with only $30 \%$ of CVST cases presenting as abnormal on plain CT. ${ }^{1}$ Systemic work-up identifies prothrombotic causes in two-thirds of patients. ${ }^{1}$ The prognosis is excellent in most patients after timely anticoagulation, which halts the propagation of CVST and thereby improves venous drainage. ${ }^{1}$

Thromboangiitis obliterans is a rare, segmental inflammatory disease of the small- to medium-sized arteries and veins of the extremities, though it may also affect cerebral vessels. ${ }^{2,3}$ The disease is characterized by highly cellular and inflammatory occlusive thrombi, with relative sparing of the vessel wall. Patients are often middle-aged, male smokers who develop distal extremity ischemia and digit ulcers. The combination of thromboangiitis obliterans and CVST is exceedingly rare.

\section{References}

1. Saposnik G, Barinagarrementeria F, Brown RD Jr, et al. Diagnosis and management of cerebral venous thrombosis: a statement for healthcare professionals from the American Heart Association/American Stroke Association. Stroke 2011;42:1158-92.

2. Bischof F, Kuntz R, Melms A, et al. Cerebral vein thrombosis in a case with thromboangiitis obliterans. Cerebrovasc Dis 1999;9:295-7.

3. Rivera-Chavarría IJ, Brenes-Gutiérrez JD. Thromboangiitis obliterans (Buerger's disease). Ann Med Surg (Lond) 2016;7:79-82.

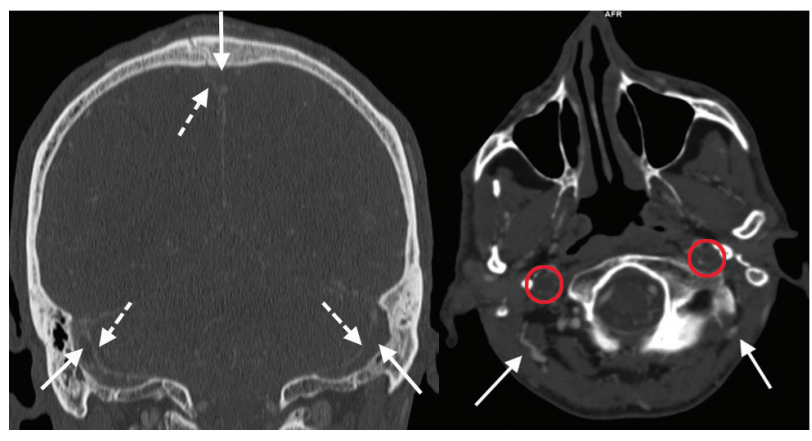

Figure 1: Computed tomography venogram from a 51-year-old man at time of presentation. The left image shows occlusion (solid arrows) of the superior sagittal sinus and both sigmoid sinuses with minimal contrast enhancement at the edges (dotted arrows). The right image shows occlusion of both internal jugular veins (circles) and venous drainage via occipital veins (arrows).

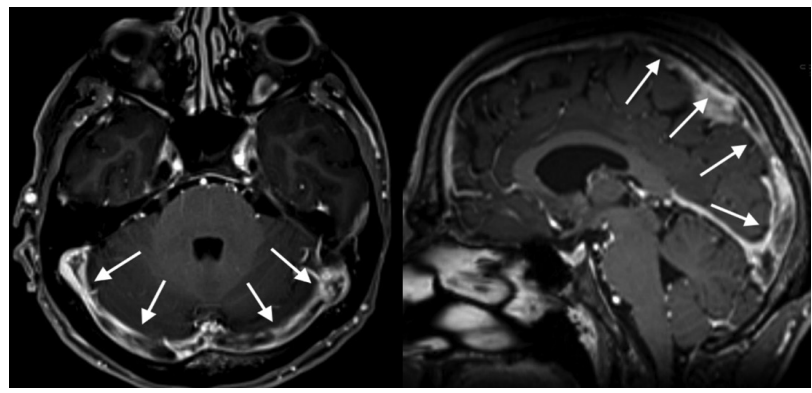

Figure 2: Magnetic resonance venogram, 15 days after patient presentation, showing persistent thrombosis of bridging veins, both internal jugular veins, the superior sagittal sinus and transverse sinuses (arrows).

\section{Competing interests: None declared.}

This article has been peer reviewed.

The authors have obtained patient consent.

Affiliations: Department of Neurology (Einsiedler, Topakian), and Institute of Radiology (Hödl), Academic Teaching Hospital WelsGrieskirchen, Wels, Austria

Content licence: This is an Open Access article distributed in accordance with the terms of the Creative Commons Attribution (CC BY-NC-ND 4.0) licence, which permits use, distribution and reproduction in any medium, provided that the original publication is properly cited, the use is noncommercial (i.e., research or educational use), and no modifications or adaptations are made. See: https://creativecommons.org/licenses/by-nc-nd/4.0/

Correspondence to: Raffi Topakian, raffi.topakian@hotmail.com 